\title{
Pouch-related fistula and intraoperative tricks to prevent it
}

\author{
F. Selvaggi - G. Pellino
}

Received: 12 November 2014/ Accepted: 12 November 2014/Published online: 4 January 2015

(C) Springer-Verlag Italia Srl 2015

Restorative proctocolectomy with ileal pouch-anal anastomosis (IPAA) is the mainstay of treatment for refractory ulcerative colitis (UC) or dysplasia in chronic UC. In spite of the advances in the medical treatment for UC, up to $30 \%$ of patients may still require surgery [1]. Irrespective of age at surgery and disease duration [2, 3], IPAA achieves optimal functional results, and this is reflected in health-related quality of life (HRQoL). Early postoperative pelvic sepsis, which is responsible for significant functional disturbances [4] and impaired HRQoL in the long term [1$6]$, is the most dreaded complication of IPAA [4, 5]. Up to $25 \%$ of IPAA patients are affected to some degree [1-7] and are at greater risk of pouch-related fistula. The risk of pelvic sepsis is inversely proportional to the surgeon's experience with pouch surgery, suggesting that some factors responsible for pelvic sepsis and late formation of pouch-related fistulae are modifiable.

Pouch-related fistulae can develop at any time, but often occur some months after IPAA, and might be related to medical factors and unidentified Crohn's disease (CD) [7, 8], but technical faults may be responsible for this complication in a relevant number of patients.

Treatment for pouch-related fistulae is difficult. The article on this type of fistula, recently published by Gaertner et al. [8] in this journal, is of great interest. The authors describe the outcomes of the treatment for pouchrelated fistula after IPAA in a retrospective series from 1989 through 2011. Twenty-five patients presented with pouch-related fistula. Each patient underwent on average 2.8 (range 1-10) procedures or repairs, consisting of seton

F. Selvaggi $(\bowtie) \cdot$ G. Pellino

Department of General Surgery, Second University of Naples,

Via F.Giordani, 42, 80122 Naples, Italy

e-mail: fselvaggi@hotmail.com drainage $(n=20)$, fibrin glue injection $(n=11)$, fistulotomy $(n=5)$, collagen plug insertion $(n=4)$, transperineal repair $(n=3)$, ileal advancement flap $(n=2)$, insertion of cutting seton $(n=2)$, Martius flap $(n=1)$, transabdominal repair $(n=1)$, and revision of ileal pouch-anal anastomosis with gracilis muscle interposition $(n=1)$. In $24 \%$ of patients, a temporary loop ileostomy was fashioned, whereas some $\mathrm{CD}$ patients received concomitant medical treatment. Complete healing was achieved in $64 \%$ of patients with a stepwise diagnostic and therapeutic approach.

The paper includes $60 \%$ of patients with CD of the pouch, which was the only factor associated with persistent fistulas in this series [8]. The authors provide their algorithm for managing ileal-pouch-related fistulas based on their etiology (surgical complications, cryptoglandular, or CD).

However, every effort should be made to reduce the incidence of surgical complications by acting on modifiable peri- and intraoperative factors.

We have further subdivided fistula etiology specific to ileoanal pouches in Table 1.

Early pelvic sepsis $[4,5]$ is mainly due to breakdown of the IPAA and may determine peri-pouch abscess formation. The clinical impact of pelvic sepsis can be reduced by a temporary stoma, which is mandatory in patients with risk factors for anastomotic complications [9]. In addition, a stoma makes it possible to treat a potential peri-pouch abscess via a transperineal approach, with no need of repeated laparotomy. Early recognition of pelvic sepsis is the key. Pelvic- and peri-pouch collections need timely treatment. The transperineal approach should be preferred when possible and certainly used in patients with diversion ileostomies, and the ideal drainage route is the line of the ileoanal anastomosis. The abscess can be drained by removing some sutures or staples. If there is a large 
Table 1 Main causes of pouch-related fistula after ileal pouch-anal anastomosis

1. Pelvic sepsis

2. Technical errors

(a) incorrect activation of the stapler, with early breakdown of the suture line, staple height too short for tissue thickness resulting in ischemia, incorporation of the posterior wall of the vagina due to suboptimal exposure and slipping of the vagina through the stapling device components

(b) faults in pouch fashioning

(c) long residual rectal muscle cuff, suboptimal $(>2-3 \mathrm{~cm}$ ) or difficult mucosectomy

(d) tension of the anastomosis, due to inadequate lengthening of the pouch to reach the pelvis

3. Chronic erosion of the metal staples(e.g. pouch-vaginal fistula)

4. Crohn's disease

abscess, a de Pezzer catheter can be left in place. A recently introduced conservative approach is to drain the cavity with an absorbent endosponge [10]. However, this is likely to result in late formation of fistula and stricture. In that case, transanal redo-anastomosis could be an option $[11,12]$.

In very low anastomoses, an adequate identification and retraction of the vagina is mandatory. This is made easier by means of St. Marks anterior retractors, pulling up the vaginal wall. In difficult cases, to prevent the posterior vaginal wall from slipping under the retractor, it could be useful to have a perineal assistant to introduce a finger through the vagina during firing. Another way to avoid incorporating the posterior vaginal wall in the stapler is to avoid manipulating the anterior vaginal wall and resect the distal rectum obliquely so that the anterior portion of the transected upper anal canal is longer than the posterior one. Resected doughnuts must be inspected intraoperatively, looking for an extra piece of tissue. The ideal solution, if extra tissue is found, is to take down and redo the anastomosis.

The most frequently used pouch is the stapled J-pouch, which is easier to fashion. After approximating two terminal ileal loops $16-20 \mathrm{~cm}$ proximally to the staple line of the terminal ileum and checking for adequate length to reach the anus (see below), an enterotomy is performed longitudinally. A linear stapler is introduced and activated, after checking that the loops' mesentery is not caught in the staple line. Two to three loads are usually needed. The suture line can then be reinforced with a running monofilament suture. When the pouch is fashioned by means of hand-sewn sutures and the anastomosis is stapled, a running suture can be used for the upper portion of the pouch body, whereas for the lower portion of the pouch body, close to the site of ileoanal anastomosis, single sutures must be used. This detail avoids breakdown of the entire continuous suture.

After the ileoanal anastomosis, the commonest site of fistulation is the blind tip of the terminal ileum, which assumes the so-called dog's ear shape when the ileum is transected flush with the cecum, at a right angle. This remnant can angulate and fistulize. In order to reduce the incidence of this complication, it can be useful to transect the ileum by angulating the stapler to form an acute angle, so that when the terminal ileum is approximated to the proximal ileal loop to be used for the pouch, it creates an oblique, lateral to medial, and caudal to cranial tip, less prone to detach from the pouch body and fistulize (Fig. 1). In addition, a stitch can be passed to tuck the blind tip to the pre-pouch ileum, just above the pouch inlet.

Before detaching the rectum from the anus, it is important to check that dissection has been carried out far enough caudad to reach the level of the levators. This can be done by asking the perineal assistant to introduce a finger through the anus to touch the operating surgeon's hand holding the rectum from above. The assistant can show the distance between the anus and the most proximal height where the surgeon's hand is touched, after removing the inserted finger. The maneuver is very useful in narrow and "deep" pelvis, and can be repeated to avoid long rectal remnant, a cause of subsequent complications. Correct mucosectomy, patient positioning, and perineal exposure, as well as the surgeon's comfort and view account for more than $90 \%$ of the success rate of the procedure. The patient's coccyx should be next to the end of the table, the legs should be moved from Lloyd-Davies to lithotomy position, and the table should be raised so that the anus is at the level of the surgeon's eyes. A mild Trendelenburg position and a head light may be useful. The Lone Star Retractor System eases the procedure, and an Eisenhammer anal dilator can be used as an alternative or an adjunct. Mucosectomy should be started after injecting adrenaline (1:200,000 plus normal saline) in the mucosa of the anorectal stump, and carried out carefully, removing all the visible residual mucosa by means of cautery or scissors. After checking that an adequate length of the vascular axis has been reached, the perineal surgeon introduces two long Klemmer forceps transanally (at 3 and 9 o'clock) into the abdomen, picking up and drawing down two sutures, which have to be placed at the inferior end of the pouch, at the 
Fig. 1 Upper drawing shows the ileum being transected flush to the cecum with a linear stapler. This causes a remnant at the pouch tip (dog's ear), which can angulate and fistulize. The lower drawing shows transection of the small bowel angulating the stapler, thus creating an oblique, lateral to medial, and caudal to cranial tip, less prone to detach from the pouch body and fistulize
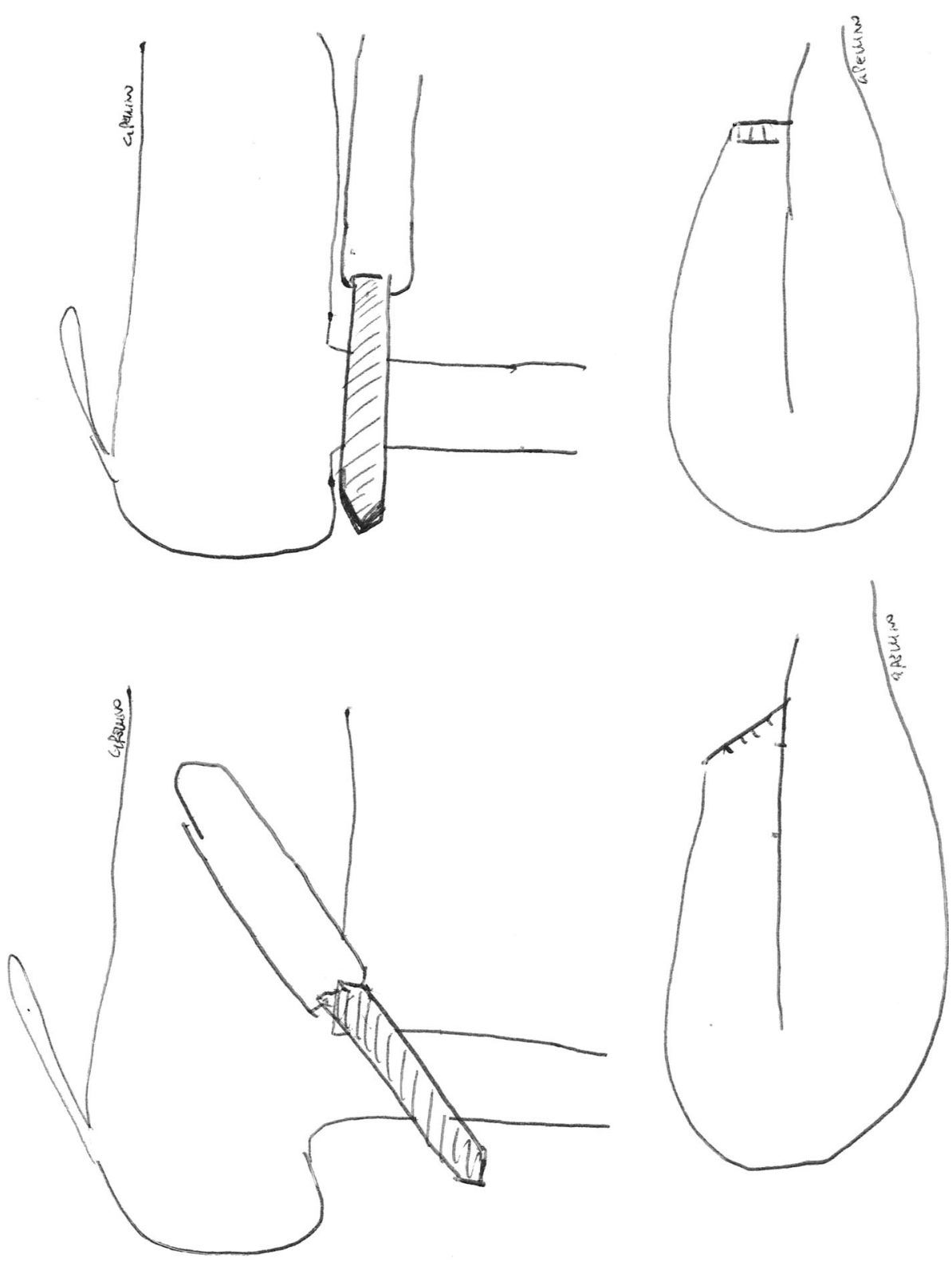

end of the abdominal procedure. It is important that the abdominal assistant carefully check at this stage that the reservoir does not twist on the vascular axis during passage through the pelvis. Right-handed surgeons should start the ileoanal anastomosis at 3 o'clock, incorporating the opened ileum delivered through the anus and should pass the stitch

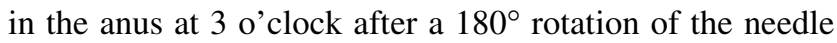
holder in the operating hand, tying the suture. The same maneuver is performed at 9 o'clock, and an Eisenhammer retractor is gently inserted through the anus into the pouch, allowing placement of stitches at 6 at 12 o'clock. Then, anastomosis is continued with interrupted absorbable $2 / 0$ sutures. Approximately 12 stitches are required. At the end of the procedure, the surgeon checks that the anastomosis is symmetric and that the stitches are close enough together.
At this time, it can be helpful to use the tip of the forceps to check that no space is left between each pair of stitches.

An anastomosis under tension is not a successful anastomosis. Tension can cause early breakdown of the anastomosis, and fistula and stricture in the long term. Obtaining an adequate length of mesentery is thus crucial for the success of the entire procedure. It is important that the removal phase of the IPAA be correctly performed by fully mobilizing the ileal mesentery, exposing the inferior border of the duodenum and the pancreas before mobilizing the colon and the rectum, and dividing the colic vessels. Pouch reach is considered adequate if the planned apex reaches the inferior border of the symphysis pubis. The "trial descent" of the pouch to the anus represents a more reliable way to directly assess the reach and should be 
performed at least in dubious cases. How can adequate length be obtained when the pouch does not reach? In our practice, we prefer to fold the pouch to lie anteriorly with the mesentery posterior, which allows us to gain $0.5-1 \mathrm{~cm}$ extra-length compared with the anterior mesentery. With several horizontal, front-and-back, relaxing incisions of the peritoneum perpendicular to the superior mesenteric artery (SMA), one can gain up to $2 \mathrm{~cm}$ (stepladder incisions). These may be particularly useful in patients with adhesions and mesenteric fibrosis due to previous open surgery. We usually divide the ileocolic artery close to the SMA, and remove the residual string of peritoneum. When reach appears inadequate, up to $3.5 \mathrm{~cm}$ can be gained by using proximal superior mesenteric pedicle division with preservation of the ileocolic pedicle rather than ileocolic division [13]. An alternative suitable for patients highly likely to have a problem with pouch reach is that described by Goes et al. [14] using the right branch of the middle colic artery. The authors estimated that extra $11 \mathrm{~cm}$ could be gained. Interposition of a vein graft to the SMA has been also described in a case of severely foreshortened mesentery [15]. Other factors affecting pouch reach are the type of anastomosis, since stapled IPAA to the upper anal canal requires $2 \mathrm{~cm}$ less reach, and pouch shape, with S-pouches reaching 1-2 cm further than J-pouches and $\mathrm{W}$-pouches.

Intraoperative endoscopy, besides inspecting the ileal pouch mucosa, height and appearance of the anastomosis, can reveal leaks in the pouch or at the IPAA. This is done by gently inflating the pouch at the level of the IPAA, and then in the pouch body, after pouring warm water into the abdomen to immerge the entire pouch. A leak is revealed by the presence of bubbles, allowing localization and direct repair. However, endoscopy should be performed cautiously, to avoid damaging the IPAA.

Stapled IPAA and placement of non-absorbable staples can result in late formation of fistula due to erosion of the sutures through the posterior wall of the vagina, especially when observed many years after IPAA, and is favored by slim patient. This factor is hardly modifiable.

$\mathrm{CD}$ is the principal cause of pouch failure in the long term, presenting with abscesses, fistulas, and strictures of the pouch body. Following up the good results achieved with combined treatment with biologics in CD fistulas [16, 17], the chances of preserving the pouch in these patients are slightly higher when recent medical treatments are used, either alone or combined with surgery [18].

Blind sinuses originating from the anastomosis often heal delaying ileostomy closure [19], but debridement and unroofing or filling with fibrin glue may be an option in some patients.

Adopting these strategies would minimize the risk of subsequent pouch-related fistula formation. However, this eventuality is not eliminated, due to unmodifiable factors as well as the possible development of cryptoglandular fistulae, and the need for evidences concerning the ideal, definitive treatment for complex pouch-related fistulae remains.

\section{Conflict of interest None.}

\section{References}

1. Ross H, Steele SR, Varma M, Standards Practice Task Force of the American Society of Colon and Rectal Surgeons et al (2014) Practice parameters for the surgical treatment of ulcerative colitis. Dis Colon Rectum 57:5-22

2. Pellino G, Sciaudone G, Miele E et al (2014) Functional outcomes and quality of life after restorative proctocolectomy in paediatric patients: a case-control study. Gastroenterol Res Pract 2014:340341

3. Pellino G, Sciaudone G, Candilio G et al (2013) Complications and functional outcomes of restorative proctocolectomy for ulcerative colitis in the elderly. BMC Surg 13(Suppl 2):S9

4. Selvaggi F, Sciaudone G, Limongelli P et al (2010) The effect of pelvic septic complications on function and quality of life after ileal pouch-anal anastomosis: a single center experience. Am Surg 76:428-435

5. Kiely JM, Fazio VW, Remzi FH, Shen B, Kiran RP (2012) Pelvic sepsis after IPAA adversely affects function of the pouch and quality of life. Dis Colon Rectum 55:387-392

6. Papadopoulos VN, Michalopoulos A, Apostolidis S (2010) Ileal pouch dysfunction. Tech Coloproctol 14(Suppl 1):S83-S85

7. Fazio VW, Kiran RP, Remzi FH et al (2013) Ileal pouch anal anastomosis: analysis of outcome and quality of life in 3707 patients. Ann Surg 257:679-685

8. Gaertner WB, Witt J, Madoff RD, Mellgren A, Finne CO, Spencer MP (2014) Ileal pouch fistulas after restorative proctocolectomy: management and outcomes. Tech Coloproctol 18:1061-1066

9. Pellino G, Sciaudone G, Canonico S, Selvaggi F (2012) Role of ileostomy in restorative proctocolectomy. World J Gastroenterol 18:1703-1707

10. Verlaan T, Bartels SA, van Berge Henegouwen MI, Tanis PJ, Fockens P, Bemelman WA (2011) Early, minimally invasive closure of anastomotic leaks: a new concept. Colorectal Dis 13(Suppl 7):18-22

11. Maslekar S, Sagar PM, Harji D, Bruce C, Griffiths B (2012) The challenge of pouch-vaginal fistulas: a systematic review. Tech Coloproctol 16:405-414

12. Fazio VW, Tjandra JJ (1992) Pouch advancement and neoileoanal anastomosis for anastomotic stricture and anovaginal fistula complicating restorative proctocolectomy. Br J Surg 79:694-696

13. Martell P, Majery N, Savigny B, Sezeur A, Gallot D, Malafosse M (1998) Mesenteric lengthening in ileoanal pouch anastomosis for ulcerative colitis: is high division of the superior mesenteric pedicle a safe procedure? Dis Colon Rectum 41:862-866

14. Goes RN, Nguyen P, Huang D, Beart RWJ (1995) Lengthening of the mesentery using the marginal vascular arcade of the right colon as the blood supply to the ileal pouch. Dis Colon Rectum 38:893-895

15. Metcalf DR, Nivatvongs S, Sullivan TM, Suwanthanma W (2008) A technique of extending small-bowel mesentery for ileal pouch-anal anastomosis: report of a case. Dis Colon Rectum 51:363-364 
16. Sciaudone G, Di Stazio C, Limongelli P et al (2010) Treatment of complex perianal fistulas in Crohn disease: infliximab, surgery or combined approach. Can J Surg 53:299-304

17. Sciaudone G, Pellino G, Riegler G, Selvaggi F (2011) Infliximab in drug-naïve patients with failed ileorectal anastomosis for Crohn's disease: a new chance for sparing the rectum? Eur Surg Res 46:163-168

18. Araki T, Okita Y, Fujikawa $\mathrm{H}$ et al (2014) Redo Ileal pouch-anal anastomosis combined with anti-TNF- $\alpha$ maintenance therapy for
Crohn's disease with pelvic fistula: report of two cases. Surg Today 44:1982-1985

19. Selvaggi F, Pellino G, Canonico S, Sciaudone G (2012) Is omitting pouchography before ileostomy takedown safe after negative clinical examination in asymptomatic patients with pelvic ileal pouch? An observational study. Tech Coloproctol $16: 415-420$ 